\title{
PRINCIPAIS DESFECHOS FATAIS EM INDIVÍDUOS CARDIOPATAS ACOMETIDOS POR COVID-19
}

\author{
Patricia Veiga Nascimento ${ }^{1}$ \\ Monneglesia Santana Lopes Cardoso ${ }^{1}$ \\ Ana Carolina Conceição Neves ${ }^{1}$
}

\author{
https://orcid.org/0000-0002-5785-6446 \\ https://orcid.org/0000-0001-9548-616X \\ https://orcid.org/0000-0002-9200-1929
}

Objetivo: identificar os principais desfechos fatais em individuos cardiopatas acometidos por COVID-19. Método: Trata-se de um estudo de natureza qualitativa, do tipo revisão integrativa da literatura, por meio dos descritores: Cardiopatias; Evolução Fatal; Infecções por Coronavírus e COVID-19, nas bases de dados: Literatura Latino-Americana e do Caribe em Ciências da Saúde (LILACS) e Medical Literature Analysis and Retrieval Sistem on-line (Medline); National Institutes of Health's National Library of Medicine (NIH/NLM) - PubMed; PubMed Central. Resultados: Dos 13 estudos incluidos após critérios de exclusão, somente oito textos na íntegra contemplaram elementos que respondiam à pergunta da pesquisa. Os mecanismos pelo quais o SARS-CoV-2 promovem lesões ao tecido miocárdico ainda não são completamente conhecidos. No entanto, acredita-se que há um risco aumentado de lesão cardíaca aguda associada à infecção mais grave por COVID-19 e essa lesão está associada ao evento fatal. Conclusão: Os achados desse estudo permitiram identificar os principais desfechos fatais em indivíduos cardiopatas acometidos por COVID-19, ademais demonstram que a atenção e o cuidado aos portadores de cardiopatias após o diagnóstico da COVID-19 precisam ser intensificados, especialmente pelo fato deste grupo de risco ter maior índice de mortalidade.

Descritores: COVID-19; Cardiopatias; Evolução fatal.

\section{MAIN FATAL OUTCOMES IN INDIVIDUALS WITH CARDIOPATHIES STRICKEN BY COVID-19}

Objective: To identify the main fatal outcomes of individuals with cardiopathies stricken by COVID-19. Method: A study of a qualitative nature, of a literature integrative review using the descriptors: Heart Diseases; Fatal Outcome; Coronavirus and COVID-19 infections, on the following databases: Latin American and Caribbean Health Sciences Literature (LILACS) and Medical Literature Analysis and Retrieval System on-line (Medline); National Institutes of Health's National Library of Medicine (NIH/ NLM) - PubMed; PubMed Central. Results: Out of the 13 studies included after the exclusion criteria, only eight texts fully contemplated elements that answered the question of the research. The mechanism through which SARS-CoV-2 caused lesions to the myocardial tissue are not yet completely known. However, it is believed that there is an increased risk of acute cardiac injury associated to a more acute infection through COVID-19 and such injury is associated to the fatal event. Conclusion: The findings of this study permit the identification of the main fatal outcomes in individuals with cardiopathies stricken by COVID-19, as well as demonstrating that the attention and care to individuals with cardiopathies after the diagnosis of the COVID-19 have to be intensified, mainly due to the fact that there is a higher mortality rate in this risk group.

Descriptors: COVID-19; Heart Diseases; Fatal outcome.

\section{PRINCIPALES RESULTADOS FATALES EN INDIVIDUOS CARDIÓPATAS ACOMETIDOS POR COVID-19}

Objetivo: identificar los principales resultados fatales en individuos cardiópatas acometido por COVID-19. Método: Se trata de un estudio de naturaleza cualitativa, del tipo revisión integrativa de la literatura, por medio de los descriptores: Cardiopatías; Resultado Fatal; Infecciones por Coronavirus y COVID-19, en las bases de datos: Literatura Latino-Americana y del Caribe en Ciencias de la Salud (LILACS) y Medical Literature Analysis and Retrieval System on-line (Medline); National Institutes of Health's National Library of Medicine (NIH/NLM) - PubMed; PubMed Central. Resultados: De los 13 estudios incluidos después de los criterios de exclusión, solamente ocho textos en la íntegra contemplaron elementos que respondian a la pregunta de la investigación. Los mecanismos por los cuales el SARS-CoV-2 promovieron lesiones al tejido miocárdico aún no son completamente conocidos. No obstante, se cree que existe un riesgo augmentado de lesión cardiaca aguda asociada a la infección más grave por COVID-19 y esa lesión está asociada al evento fatal. Conclusión: Los hallazgos de esa investigación permitieron identificar los principales resultados fatales en individuos cardiópatas acometidos por COVID-19, además demostraron que la atención y el cuidado a los portadores de cardiopatías después del diagnóstico del COVID-19 necesitan ser intensificados, especialmente por el hecho de ese grupo de riesgo tener mayor índice de mortalidad.

Descriptores: COVID-19; Cardiopatías; Resultado fatal. 


\section{INTRODUÇÃO}

Em dezembro de 2019, um conjunto de casos de pneumonia viral inexplicável foi reportado em Wuhan, na China. Dando início à busca pela identificação do agente etiológico responsável, um novo coronavírus foi sequenciado e nomeado como SARS-CoV-2 (do inglês: Severe Acute Respiratory Syndrome Coronavirus 2) ${ }^{1}$. Este novo coronavírus causa a Coronavirus Disease 2019 (COVID-19), uma doença classificada pela OMS, em 11 de março de 2020, como uma pandemia ${ }^{2}$. A alta transmissibilidade do vírus tem provocado mais de três milhões de casos mundialmente, desses mais 80 mil casos foram confirmados no Brasil, onde já provocou mais de cinco mil óbitos, com uma taxa de letalidade a $7,0 \%{ }^{3-4}$

O quadro clínico da COVID-19 é semelhante ao de outras viroses respiratórias, com febre, tosse, geralmente seca, cansaço e, em casos mais graves, dispnéia, sangramento pulmonar, linfopenia grave e insuficiência renal. Em $80 \%$ dos casos, os sintomas são leves. O diagnóstico dos casos sintomáticos deve ser confirmado com a pesquisa do vírus por reação em cadeia da polimerase (PCR) de swab nasal ${ }^{5}$.

Sabe-se que a população brasileira vive uma transformação caracterizada pelo aumento percentual da população de idosos gerando um predomínio de doenças crônicas, dentre esses distúrbios, os acometimentos cardiovasculares $^{6}$. As doenças cardiovasculares (DCV) prevalecem como a principal causa de óbitos no mundo e fazem parte dos fatores associados ao desfecho clínico da internação dos idosos em Unidades de Terapia Intensiva (UTI). Um estudo mostrou que as doenças cardiovasculares foram as mais incidentes dentre as doenças crônicas não transmissiveis, atingindo $68,7 \%$ dos idosos admitidos em $\mathrm{UTI}^{7}$.

Pesquisa publicada pelo Centro Chinês de Controle e Prevenção de Doenças, com dados de 44.672 casos confirmados da COVID-19, relatou mortalidade de 2,3\% e as comorbidades mais frequentes com desfechos fatais foram hipertensão arterial, diabetes mellitus, doença cardiovascular e idade acima de 70 anos $^{7}$. Dados recentes da pandemia da COVID-19 descrevem um número considerável de casos, sendo as principais manifestações relacionadas ao sistema cardiovascular e sua prevalência: arritmias (16\%); isquemia miocárdica (10\%); miocardite $(7,2 \%)$ e choque (1-2\%) ${ }^{8}$.

Vale a pena ressaltar que os indivíduos graves admitidos nas UTI chinesas, evoluíram com hipoxemia e síndrome de desconforto respiratório entre o nono e o 12으 dia. Os pacientes que foram a óbito apresentaram complicações cardiovasculares, como choque e disfunção ventricular entre o 14 으 e o 20 으 dia da infecção ${ }^{8}$.

Considerando a importância clínica e social das doen- ças cardiovasculares no Brasil, diante do atual contexto de pandemia e do potencial de letalidade do vírus, em pacientes com alguma cardiopatia, este estudo tem como objetivo identificar os principais desfechos fatais em indivíduos cardiopatas acometidos por COVID-19.

\section{MÉTODO}

\section{Tipo de estudo}

Trata-se de um estudo de natureza qualitativa, do tipo revisão integrativa (RI) da literatura, desenvolvida com a finalidade de reunir e sintetizar resultados de pesquisas sobre um determinado tema ou questão, de maneira sistemática e ordenada, com o intuito de aprofundar o conhecimento do tema investigado 9

O delinear do estudo se deu por meio de seis etapas assim descritas: 1) elaboração da questão norteadora; 2) busca de literatura/ estabelecimento de critérios para a inclusão e exclusão de estudos; 3) definição das informações a serem extraídas dos estudos selecionados; 4) análise crítica dos estudos incluídos; 5) interpretação dos resultados; 6) apresentação da revisão/ sintese do conhecimento em quadro resumo ${ }^{10}$

A questão norteadora desse estudo é: "quais são os principais desfechos fatais em individuos com cardiopatia prévia ao ser acometido pela COVID-19?". A escolha dos descritores se baseou na seleção dos termos inseridos nos descritores em Ciências da Saúde da Biblioteca Virtual em Saúde (DeCS-BVS): Cardiopatias/Heart Diseases; Evolução Fatal/Fatal Outcome; Infecções por Coronavirus/Coronavirus Infections (DeCS) e Medical Subject Headings (MeSH-NLM): COVID-19. Para a busca utilizou-se o operador booleano AND para relacionar os descritores procurados na pesquisa avançada de cada base.

\section{Coleta de dados}

A coleta de dados ocorreu no mês de abril de 2020, nas seguintes bases de dados: Literatura Latino-Americana e do Caribe em Ciências da Saúde (LILACS) e Medical Literature Analysis and Retrieval Sistem on-line (Medline); National Institutes of Health's National Library of Medicine (NIH/ NLM) - PubMed; PubMed Central ${ }^{\circledR}(P M C)$.

Definiram-se como critérios de inclusão as produções científicas a partir de 2019, com artigos completos, online e disponiveis gratuitamente, publicados nos idiomas inglês e português. Como critérios de exclusão, os artigos repetidos, disponíveis em idioma divergente dos citados, não disponiveis na íntegra e aqueles que não responderam à questão norteadora da revisão.

A combinação dos descritores resultou em 72 publicações e, com a aplicação dos critérios de inclusão e exclusão, foram selecionados 13 artigos para análise crítica, conforme Figura 1 . 
Figura 1 - Representação esquemática da síntese e análise dos resultados. 2020.

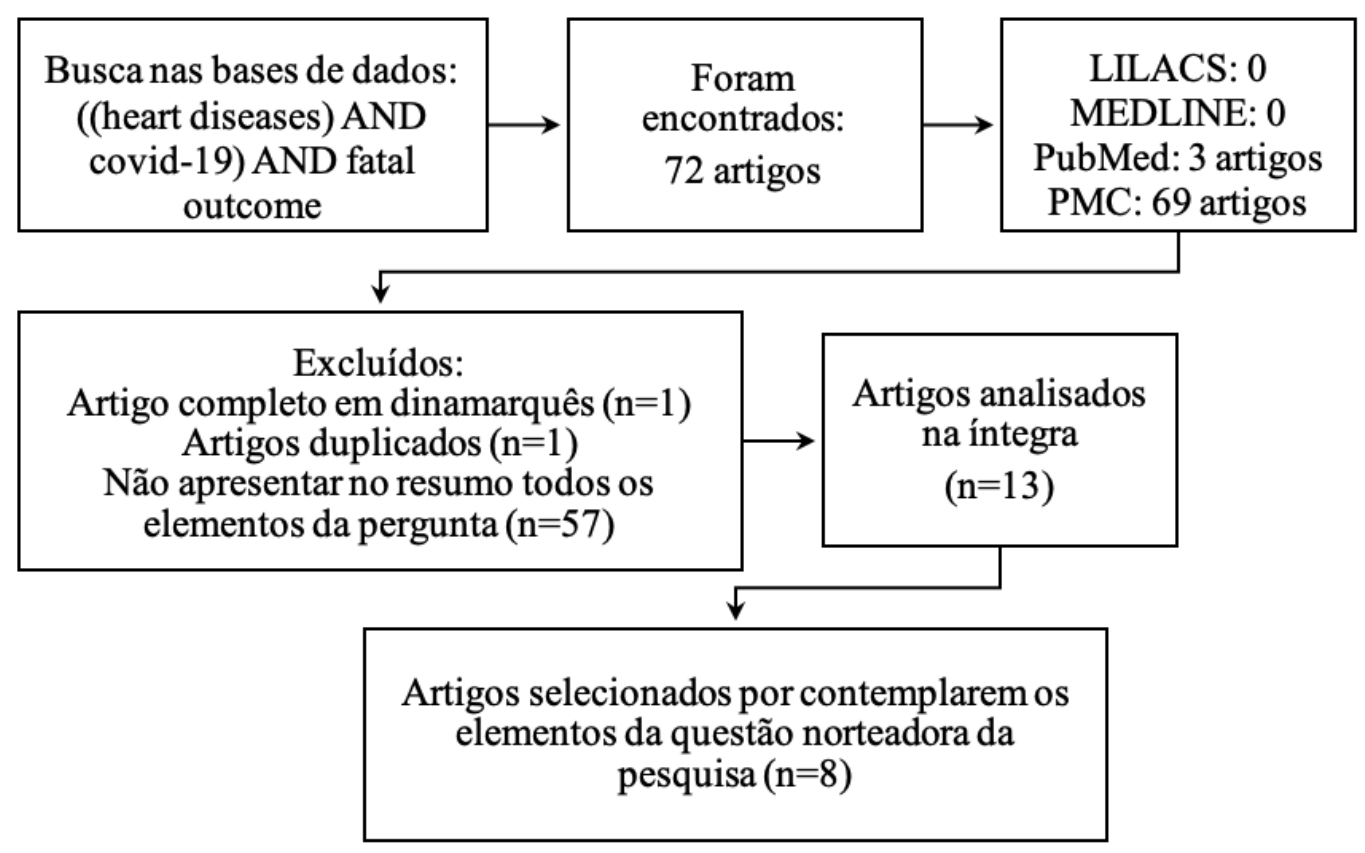

Análise dos dados

Procedeu-se à interpretação e discussão dos resultados, destacando-se os trabalhos que trouxeram maior contribuição para responder ao problema da pesquisa. A próxima etapa constitui-se na apresentação, revisão e síntese dos dados encontrados, capazes de fornecer informações sobre os principais desfechos fatais em pessoas com cardiopatias.

\section{Aspectos éticos}

No presente estudo a coleta de dados envolveu dados secundários e públicos, disponíveis em bases de dados online. No entanto, é importante salientar que foram respeitados os preceitos éticos inerentes ao desenvolvimento de qualquer pesquisa e, para esse estudo, foram respeitados na íntegra os conteúdos das produções científicas aqui utilizadas, assim como todos os direitos autorais, com base na Lei de Direitos Autorais no 9.610, de 19 de fevereiro de $1998^{11}$.

Quadro 1 - Caracterização dos estudos selecionados. 2020.

\section{RESULTADOS}

Dos 13 estudos incluídos após critérios de exclusão, somente oito textos na íntegra contemplaram elementos que respondiam à pergunta da pesquisa. O Quadro 1 mostra a caracterização dos estudos selecionados. As publicações de um a sete foram encontradas na base de dados da PMC, enquanto o artigo oito localizava-se no PubMed. Todos os textos incluídos foram publicados na língua inglesa e no ano de 2020.

Após leitura flutuante, cinco estudos não foram considerados para constituir o corpus deste estudo, pois apesar de apresentarem literatura compativel com o tema, os mesmos não traziam elementos capazes de responder à questão norteadora.

\begin{tabular}{|c|c|c|c|}
\hline Título & Autores/ Revista & Objetivo & Tipo de estudo/Local \\
\hline $\begin{array}{c}\text { Elevated troponin in patients } \\
\text { with Coronavirus Disease } \\
2019 \text { (COVID-19): possible } \\
\text { mechanisms }\end{array}$ & $\begin{array}{c}\text { Tersalvi G, Vicenzi M, Cala- } \\
\text { bretta D, Biasco L, Pedrazzini } \\
\text { G, Winterton D.Journal of } \\
\text { Cardiac Failure }\end{array}$ & $\begin{array}{c}\text { Explorar as evidências dis- } \\
\text { poniveis sobre a associação } \\
\text { entre COVID-19 e lesão } \\
\text { miocárdica. }\end{array}$ & Revisão \\
\hline $\begin{array}{c}\text { Toward understanding the } \\
2019 \text { Coronavirus and its } \\
\text { impact on the heart }{ }^{13}\end{array}$ & $\begin{array}{c}\text { Becker RC. } \\
\text { Journal of Thrombosis and }\end{array}$ & $\begin{array}{c}\text { Elaborado para resumir nos- } \\
\text { sa compreensão atual do } \\
\text { SARS-CoV-2 e seus efeitos } \\
\text { no coração. }\end{array}$ & Breve editorial \\
\hline
\end{tabular}




\begin{tabular}{|c|c|c|c|}
\hline $\begin{array}{l}\text { Cardiovascular Implications } \\
\text { of Fatal Outcomes of Pa- } \\
\text { tients with Coronavirus Dis- } \\
\text { ease } 2019 \text { (COVID-19) }{ }^{14}\end{array}$ & $\begin{array}{l}\text { Guo T, Fan Y, Chen M, Wu X, } \\
\text { Zhang L, He T et al. } \\
\text { JAMA Cardiology }\end{array}$ & $\begin{array}{c}\text { Avaliar a associação de } \\
\text { doença cardiovascular sub- } \\
\text { jacente (DCV) e lesão do } \\
\text { miocárdio com resultados } \\
\text { fatais em pacientes com } \\
\text { COVID-19. }\end{array}$ & $\begin{array}{l}\text { Estudo observacional re- } \\
\text { trospectiva/ Wuhan - China }\end{array}$ \\
\hline $\begin{array}{l}\text { Cardiovascular disease and } \\
\text { COVID-1917 }\end{array}$ & $\begin{array}{l}\text { Bansal M. Diabetes and Me- } \\
\text { tabolic Syndrome }\end{array}$ & $\begin{array}{l}\text { Fornece uma visão geral de } \\
\text { várias manifestações CV em } \\
\text { pacientes que se apresen- } \\
\text { tam com COVID-19. }\end{array}$ & Revisão \\
\hline $\begin{array}{l}\text { Prevalence and impact of } \\
\text { cardiovascular metabolic } \\
\text { diseases on COVID-19 in } \\
\text { China }^{18}\end{array}$ & $\begin{array}{l}\text { Li B, Yang J, Zhao F, Zhi L, } \\
\text { Wang X, Liu L et al. } \\
\text { Clinical Research in Cardi- } \\
\text { ology }\end{array}$ & $\begin{array}{l}\text { Identificar as características } \\
\text { epidemiológicas e clínicas } \\
\text { cardiovasculares da infec- } \\
\text { ção 2019-nCoV com maior } \\
\text { precisão e desvendar o im- } \\
\text { pacto da infecção na lesão } \\
\text { cardiaca. }\end{array}$ & Meta-análise \\
\hline $\begin{array}{c}\text { Clinical Features of } 85 \text { Fatal } \\
\text { Cases of COVID-19 from } \\
\text { Wuhan: A Retrospective } \\
\text { Observational Study }{ }^{19}\end{array}$ & $\begin{array}{c}\text { Du Y, Tu L, Zhu P, Mu M, } \\
\text { Wang R, Yang P et al. } \\
\text { Am J Respir Crit Care Med }\end{array}$ & $\begin{array}{c}\text { Relatar as características } \\
\text { clínicas de } 85 \text { casos fatais } \\
\text { com COVID-19 em dois hos- } \\
\text { pitais em Wuhan. }\end{array}$ & $\begin{array}{c}\text { Estudo observacional re- } \\
\text { trospectiva/ } \\
\text { Wuhan - China }\end{array}$ \\
\hline
\end{tabular}

\section{DISCUSSÃO}

Os estudos publicados até o momento têm evidenciado que a taxa geral de mortalidade por COVID-19 é de 2,3\%, entretanto, atinge 10,5\% em pacientes com DCV subjacente $\mathrm{e}^{14,17}$. Tal evidência justifica-se pelo fato de, nos pacientes com comorbidades cardiovasculares, a doença viral pode danificar de maneira mais acentuada as células do miocárdio, por meio de vários mecanismos, que geram danos diretos pelo vírus, respostas inflamatórias sistêmicas, placa coronária desestabilizada e hipóxia agravada. Por conta disso, pacientes com DCV estão mais propensos a sofrer lesão miocárdica após infecção por COVID-19 e apresentam maior risco de morte ${ }^{12,16}$.

Os mecanismos pelo quais o SARS-CoV-2 promovem le- sões ao tecido miocárdico ainda não são completamente conhecidos. Todavia, os estudos disponíveis permitem elucidar algumas hipóteses: sabendo que a ligação do SARS-CoV-2 à enzima conversora da angiotensina II (ECA-2) é uma etapa necessária para a entrada do vírus e, consequentemente, expressão fenotípica da doença, a expressão positiva de ECA-2 no sequenciamento de RNA em células do miocárdio, pode ser a chave para um melhor entendimento de lesão cardiaca, insuficiência cardiaca, arritmias e choque circulatório na COVID-1913.16

Outras hipóteses estão relacionadas ao nível sérico de troponina I cardíaca (cTnI) em pacientes com cardiopatias, principalmente hipertensão, doença coronariana e cardiomiopatia, sendo este marcador bioquímico preditor de risco 
para menor sobrevida, até mesmos nos casos de apresentação leve da COVID-1914,15. Assim, os estudos foram analisados a partir dessas duas perspectivas para identificar os principais desfechos fatais em cardiopatas acometidos por COVID-19.

Estudos que fizeram análise clínica de marcadores bioquímicos evidenciam que, níveis elevados de troponina são frequentes em pacientes com COVID-19 e significativamente associados a resultados fatais. Vários mecanismos podem explicar esse fenômeno: miocardite viral, dano miocárdico causado por citocinas, microangiopatia e Doença Arterial Coronariana (DAC) não mascarada ${ }^{12}$. No entanto, pode-se analisar também que $16 \%$ dos pacientes com DCV subjacente, mas com niveis normais de troponina, tiveram um resultado relativamente favorável ${ }^{14}$

Portanto, acredita-se que há um risco aumentado de lesão cardiaca aguda associada à infecção mais grave por COVID-2019 e essa lesão está associada ao evento fatal. Pacientes com histórico de hipertensão parecem sofrer mais com danos cardíacos ${ }^{15}$.

Pacientes com DAC preexistente e aqueles com fatores de risco para DCV aterosclerótica têm um risco aumentado de desenvolver uma sindrome coronariana aguda (SCA) durante infecções agudas, como demonstrado anteriormente em estudos epidemiológicos e clínicos de influenza e outras condições inflamatórias agudas. Isso ocorre porque há maior prevalência de troponina elevada entre pacientes com COVID-19 com DCV prévia ${ }^{12}$

A partir dos estudos analisados permite-se elucidar maiores desfechos fatais pela COVID-19 associados a pacientes com cardiopatias, como: arritmias malignas $13-16,18,19$; parada cardíaca $^{16,19}$, parada respiratória ${ }^{19}$; falência múltipla de órgãos ${ }^{16,19}$; choque séptico ${ }^{13,15,18,19}$; síndrome coronariana aguda19; coagulação intravascular disseminada ${ }^{19}$; infecção bacteriana ${ }^{18}$; lesão renal aguda ${ }^{18}$; sindrome do desconforto respiratório agudo ${ }^{18}$; anormalidades das enzimas hepáticas ${ }^{18}$; insuficiência cardíaca ${ }^{18}$

\section{Limitações do estudo}

Considera-se como limitações para este estudo o grande número de publicações imediatas no momento da pandemia, que inviabiliza tempo para maior rigor metodológico-científico; estudos de centro único, com amostras pequenas, que impossibilitam números estatisticamente significantes, capazes de ajudar na aplicação clínica.

\section{Contribuições para a prática}

Acredita-se que os resultados desse estudo podem contribuir para atuação dos profissionais de saúde, de maneira geral, devido aos resultados que evidenciam o potencial de letalidade da COVID-19 nos pacientes com algum tipo de cardiopatia. Ao elencar os principais desfechos fatais, permite que as medidas de prevenção possam ser melhor planejadas, considerando a realidade das doenças cardiovasculares no Brasil, e que as situações clínicas sejam conduzidas com maior rigor, além de promover dados para atualização de protocolos que minimizem o número de casos que evoluam para óbito.

\section{CONCLUSÃO}

Os achados desse estudo permitiram identificar os principais desfechos fatais em indivíduos cardiopatas acometidos por COVID-19, demonstrando que a atenção e o cuidado aos portadores de cardiopatias, após o diagnóstico da COVID-19, precisam ser intensificados, especialmente pelo fato de este grupo de risco ter maior índice de mortalidade.

As evidências atuais já revelam a necessidade de atenção especial aos pacientes dos grupos de risco e a importância do manejo adequado das complicações cardiovasculares.

Há um limitado número de estudos originais, entretanto, vale destacar o empenho dos profissionais que atuam na linha de pesquisa e de enfrentamento à pandemia, por estarem compartilhando, de forma precoce, resultados e experiências, auxiliando nas condutas clínicas.

Contribuições dos Autores: Neves ACC: concepção e/ou desenho do estudo, análise e interpretação dos dados, redação do artigo, revisão crítica, revisão final. Nascimento PV e Cardoso MSL: análise e interpretação dos dados, redação do artigo, revisão crítica, revisão final.

\section{REFERÊNCIAS}

1. Sun Y, Dong Y, Wang L, Xie H, Li B, Chang C et al. Characteristics and prognostic factors of disease severity in patients with COVID-19: The Beijing experience. J Autoimmun. 2020 [cited 2020 Apr 27]; 102473: In press. Available from: https://www. ncbi.nlm.nih.gov/pmc/articles/PMC7180376/.

2. Cespedes MS, Souza JCRP. SARS-CoV-2: uma revisão para o clínico. Health Sciences. 2020 [cited 2020 Apr 26]. In press.
Available from: https://preprints.scielo.org/index.php/scielo/ preprint/view/26/41.

3. Ministério da Saúde (BR). Painel Coronavirus Brasil [Internet]. 2020 [cited 2020 Apr 26]. Available from: https://covid.saude.gov.br/.

4. World Health Organization. Coronavirus disease (COVID-2019) situation reports - 101. 2020 [cited 2020 Apr 30]. Available from: https://www.who.int/docs/defaultsource/coronaviruse/situation-reports/20200430-sitrep- 
101-covid-19.pdf?sfvrsn=2ba4e093_2.

5. Costa IBSS, Bittar CS, Rizk SI, Araújo Filho AE, Santos KAQ, Machado TIV et al. The Heart and COVID-19: What Cardiologists Need to Know. Arq Bras Cardiol. 2020 [cited 2020 Apr 30]; 114(5):805-816. Available from: http:// publicacoes.cardiol.br/portal/abc/portugues/aop/2020/ AOP_2020-0279.pdf.

6. Silva TTM, Dantas RAN, Dantas DV, Lima MSM, Alves LCM, Costa ICS, et al. Pacientes submetidos à angioplastia transluminal coronariana: análise epidemiológica e angiográfica. Rev. Enferm. Foco [Internet]. 2019 [cited 2020 Apr 30]; 10(3):126-133. Available from: http://revista.cofen. gov.br/index.php/enfermagem/article/view/1991/566.

7. Simão LTSS, Lages LP, Paiva MHP, Ribeiro NLS, Araújo ERM, Leão GM. Perfil dos idosos com doenças crônicas não transmissiveis internados em unidade de terapia intensiva. Rev. Enferm. Foco [Internet]. 2019 [cited 2020 Apr 28];10(1):7680. Available from: http://revista.cofen.gov.br/index.php/ enfermagem/article/view/1329.

8. Sociedade Brasileira de Cardiologia. SBC Informa - Nota de esclarecimento. Infecção pelo Coronavírus 2019 (COVID-19). 13 de março de 2020 [cited 2020 Apr 28]. Available from: http:// www.cardiol.br/sbcinforma/2020/20200313-comunicadocoronavirus.html.

9. Mendes KDS, Silveira RCCP, Galvão CM. Revisão integrativa: método de pesquisa para a incorporação de evidências na saúde e na enfermagem. Texto contexto - enferm. [Internet]. 2008 [cited 2020 Apr 25]; 17(4):758-764. Available from: http:// dx.doi.org/10.1590/S0104-07072008000400018.

10. Sousa LMM, Vieira CMAM, Severino SSP, Antunes AV. A metodologia de revisão integrativa da literatura em enfermagem. Rev Invest Enferm. 2017 [cited 2020 Apr 25]; 17-26. Available from: https://www.researchgate. net/publication/321319742_Metodologia_de_Revisao_ Integrativa_da_Literatura_em_Enfermagem.

11. Ministério da Saúde (BR). Lei no 9.610, de 19 de fevereiro de 1998. Altera, atualiza e consolida a legislação sobre direitos autorais e dá outras providências. Diário Oficial da União. Brasília: Ministério da Saúde, 1998 [cited 2020 Apr 25]. Available from: http://www.planalto.gov.br/ ccivil_03/leis/19610.htm\#: :text=LEI\%20N\%C2\%BA\%20 $9.610 \% 2$ C $\% 20$ DE $\% 2019 \% 2$ ODE $\% 2$ OFEVEREIRO $\% 20$ DE\%201998. \&text=Altera\%2C\%20atualiza\%20e\%20 consolida\%2Oa,autorais\%20e\%20d\%C3\%Al\%20outras\%20 provid\%C3\%AAncias.\&text=Art.,os\%20que\%20lhes $\% 20$ s\%C3\%A3o\%20conexos.

12. Tersalvi G, Vicenzi M, Calabretta D, Biasco L, Pedrazzini G, Winterton D. Elevated troponin in patients with Coronavirus Disease 2019 (COVID-19): possible mechanisms. J Card Fail. 2020 [cited 2020 Apr 27]; S1071-9164(20):30357-2. Available from: https://www.ncbi.nlm.nih.gov/pmc/articles/ PMC7166030/\#!po=8.33333.

13. Becker RC. Toward understanding the 2019 Coronavirus and its impact on the heart. J Thromb Thrombolysis. 2020 [cited 2020 Apr 27]; 15:1 10. Available from: https://www.ncbi. nlm.nih.gov/pmc/articles/PMC7156795/\#_ffn_sectitle.
14. Guo T, Fan Y, Chen M, WuX, Zhang L, HeT et al. Cardiovascular Implications of Fatal Outcomes of Patients with Coronavirus Disease 2019 (COVID-19). JAMA Cardiol. 2020 [cited 2020 Apr 27]: e201017. Available from: https://www.ncbi.nlm.nih.gov/ pmc/articles/PMC7101506/.

15. Li JW, Han TW, Woodward M, Anderson CS, Zhou H, Chen YD et al. The impact of 2019 novel coronavirus on heart injury: A systemic review and Meta-analysis. Prog in Cardiovasc Dis. 2020 [cited 2020 Apr 27]: S0033-0620(20):30080-3. Available from: https://www.ncbi.nlm.nih.gov/pmc/articles/ PMC7160645/.

16. Wang L, He W, Yu X, Hu D, Bao M, Liu H et al. Coronavirus disease 2019 in elderly patients: Characteristics and prognostic factors based on 4-week follow-up. J Infect. 2020 [cited 2020 Apr 27]; 80(6):639-645. Available from: https://www.ncbi.nlm. nih.gov/pmc/articles/PMC7118526/.

17. Bansal M. Cardiovascular disease and COVID-19. Diabetes Metab Syndr. 2020 [cited 2020 Apr 27]; 14(3):247 250 Available from: https://www.ncbi.nlm.nih.gov/pmc/articles/ PMC7102662/.

18. Li B, Yang J, Zhao F, Zhi L, Wang X, Liu L et al. Prevalence and impact of cardiovascular metabolic diseases on COVID-19 in China. Clin Res Cardiol. 2020 [cited 2020 Apr 26]; 109(5):531538. Available from: https://www.ncbi.nlm.nih.gov/pmc/ articles/PMC7087935/\#CR20.

19. Du Y, Tu L, Zhu P, Mu M, Wang R, Yang P et al. Clinical Features of 85 Fatal Cases of COVID-19 from Wuhan: A Retrospective Observational Study. Am J Respir Crit Care Med. 2020 [cited 2020 Apr 28]; 201(11):1372-1379. Available from: https://www. ncbi.nlm.nih.gov/pubmed/32242738.

20. Guan WJ, Liang WH, Zhao Y, Liang HR, Chen ZS, Li YM et al. Comorbidity and its impact on 1590 patients with Covid-19 in China: A Nationwide Analysis. Eur Respir J. 2020 [cited 2020 Apr 28]; 55(5):2000547. Available from: https://www.ncbi.nlm. nih.gov/pmc/articles/PMC7098485/\#.

21. Vaduganathan M, Vardeny O, Michel T, McMurray JJV, Pfeffer MA, Solomon SD. Renin-Angiotensin-Aldosterone System Inhibitors in Patients with Covid-19. N Engl J Med. 2020 [cited 2020 Apr 28]; 382(17):1653 1659. Available from: https://www.ncbi.nlm.nih.gov/pmc/articles/PMC7121452/.

22. Vlachakis PK, Tentolouris A, Tousoulis D, Tentolouris N. Current data on the cardiovascular effects of COVID-19. Hellenic J Cardiol. 2020 [cited 2020 Apr 28]; S11099666(20):30063-4. Available from: https://www.ncbi.nlm.nih. gov/pmc/articles/PMC7165281/.

23. Maisch B. SARS-CoV-2 as potential cause of cardiac inflammation and heart failure. Is it the virus, hyperinflammation, or MODS? Herz. 2020 [cited 2020 Apr 28]: 1-2. Available from: https://www.ncbi.nlm.nih.gov/pmc/ articles/PMC7175831/.

24. Chen R, Liang W, Jiang M, Guan W, Zhan C, Wang T et al. Risk factors of fatal outcome in hospitalized subjects with coronavirus disease 2019 from a nationwide analysis in China. Chest. 2020 [cited 2020 Apr 28]; S0012-3692(20):30710-8. Available from: https://www.ncbi.nlm.nih.gov/pmc/articles/ PMC7158802/\#!po=41.6667. 